\title{
Non-destructive measurement of chlorophyll $b$ : a ratios and identification of photosynthetic pathways in grasses by reflectance spectroscopy
}

\author{
Katharina Siebke $\mathrm{A}, \mathrm{B}^{\mathrm{B}}$ and Marilyn C. Ball ${ }^{\mathrm{A}, \mathrm{C}}$ \\ ADivision of Plant Sciences, Research School of Biology, Australian National University, Canberra, \\ ACT 0200, Australia. \\ ${ }^{B}$ Present address: Heinz Walz GmbH, Eichenring 6, 91090 Effeltrich, Germany. \\ ${ }^{\mathrm{C} C}$ Corresponding author. Email: marilyn.ball@anu.edu.au
}

This paper originates from a presentation at the 1st International Plant Phenomics Symposium, Canberra, Australia, April 2009.

\begin{abstract}
Equations for non-destructive determination of chlorophyll $b: a$ ratios in grasses were developed from reflectance spectra of intact leaves of barley (Hordeum vulgare L.) and two barley mutants: clorina f2, which lacks chlorophyll $b$ and clorina f104, which has a low chlorophyll $b$ content. These plants enabled separation of effects of chlorophyll composition on reflectance spectra due to differential light absorption by chlorophylls $a$ and $b$ and to measure the effects of chlorophyll $b$ on the contribution of fluorescence emitted by chlorophyll $a$ to the reflectance spectra. Indices developed from these data were then tested on growth chamber-grown leaves from six $\mathrm{C}_{3}$ and $17 \mathrm{C}_{4}$ grass species (7 NAD-ME and 10 NADP-ME subtypes). We used the chlorophyll $b: a$ ratio because the data were less skewed than the chlorophyll $a: b$ ratio. The best index for determination of the chlorophyll $b: a$ ratio utilised wavelengths affected by chlorophyll absorbance: $\left[\mathrm{R}_{626}-0.5\left(\mathrm{R}_{603}+\mathrm{R}_{647}\right)\right] /\left[\mathrm{R}_{552}-\mathrm{R}_{626}\right]$. The chlorophyll $b: a$ ratio was significantly lower in the $\mathrm{C}_{4}$ than $\mathrm{C}_{3}$ grasses, but was not sufficient in itself to separate these two functional groups. However, because of differences in fluorescence characteristics, $\mathrm{C}_{3}$ and $\mathrm{C}_{4}$ species could be distinguished by an index based on wavelengths affected by chlorophyll fluorescence: [ $\mathrm{R}_{696}$ to $709 / \mathrm{R}_{545}$ to 567$]$.
\end{abstract}

Additional keywords: $\mathrm{C}_{3}$-photosynthesis, $\mathrm{C}_{4}$-photosynthesis, leaf pigments, remote sensing.

\section{Introduction}

Reflectance spectra are used for many tasks in remote sensing such as mapping of plant species, estimating productivity or identifying plants subject to stresses. There is much interest in remote measurement of chlorophylls $a$ and $b$, but development of equations is difficult. Variation in the content of chlorophylls $a$ and $b$ necessarily also involves changes in other physiological aspects concerning thylakoid structure and pigment composition, which could affect the reflectance spectra. In higher plants, chlorophyll $a$ is associated with the core proteins of both photosystems (PS), the inner antennae and the light harvesting complexes (LHC), whereas chlorophyll $b$ occurs only in the LHC. The chlorophyll $b$ content in light harvesting complex I (LHCI) is lower than in light harvesting complex II (LHCII). Other pigments are also differentially distributed in the photosynthetic machinery. Specifically, the core proteins of the PS and the inner antennae contain $\beta$-carotene, and the LHC contain structural as well as protective carotenoids (Paulsen 1995; Green and Durnford 1996).

Mutants may provide an ideal means of testing effects of variation in chlorophyll composition on reflectance spectra from intact leaves. For example, the photosynthetic characteristics of barley (Hordeum vulgare L.) mutants differing in contents of chlorophyll $a$ and $b$ have been well characterised (Knoetzel and Simpson 1991; Bossman et al. 1997; Gilmore et al. 2000). The clorina f104 mutant has lower amounts of both LHCI and LHCII proteins resulting in less chlorophyll $b$ than in the wild type. The clorina $f 2$ mutant lacks chlorophyll $b$ and hence, the content of LHCII proteins is much lower than in the wild type. The absence or reduction of LHC proteins also affects the content and composition of carotenoids in the mutant leaves (Harrison Nemson and Melis 1993). Furthermore, the absence or reduction of LHCII affects the chlorophyll $a$ fluorescence emission spectrum of mutant leaves (Gilmore et al. 2000).

The chl $a: b$ ratio is also expected to vary between species using $\mathrm{C}_{3}$ or $\mathrm{C}_{4}$ photosynthetic pathways (Hatch 1987). In $\mathrm{C}_{4}$ grasses, the gas-tight bundle-sheath cells contain less PSII than mesophyll cells (Edwards and Walker 1983; Hatch 1987; Ghannoum et al. 2005). This has the advantage that the accumulation of oxygen is prevented in bundle sheath cells while the special energy requirements for $\mathrm{C}_{4}$ photosynthesis can be achieved by the remaining PSI (Hatch 1987). The lack 
of PSII in the bundle sheaths is more complete in NADP-ME than NAD-ME type grasses. For the mesophyll cells, Ghannoum et al. 2005 observed a relatively high amount of both PS per chlorophyll, particularly in the NADP-ME type grasses. Because of these biochemical differences between bundle sheath and mesophyll cells, there is a tendency for leaves of $\mathrm{C}_{4}$ grasses to contain more PSI relative to PSII than leaves of $\mathrm{C}_{3}$ grasses. It follows that the chl $a: b$ ratio would also be higher in $C_{4}$ than $C_{3}$ grasses (Hatch 1987).

One objective of the present study was to find a spectral method for determination of the ratio of chlorophyll $b$ to chlorophyll $a$ (chl $b: a$ ratio) in grass leaves. This ratio was used for two reasons. First, the distribution was less skewed than chl $a: b$, as needed for statistical analyses. Second, as we used mutants with no $\operatorname{chl} b$, the chl $a: b$ ratio would become undefined, again creating difficulties for analyses. We measured reflectance spectra of barley wild type and two barley mutants, clorina f104 and clorina f2. We used these mutants because they have large variation in chlorophyll $b$ with relatively low correlation to chlorophyll $a$, at least within our dataset. Indices developed from the reflectance spectra of the barley plants were then tested on a set of six $\mathrm{C}_{3}$ and $17 \mathrm{C}_{4}$ grass species, with the latter including seven NAD-ME and 10 NADP-ME type species. The spectra of the $\mathrm{C}_{3}$ and $\mathrm{C}_{4}$ grass species were also used to achieve the second objective to find an index that could distinguish between grasses using either $\mathrm{C}_{3}$ or $\mathrm{C}_{4}$ photosynthetic pathways.

\section{Materials and methods}

\section{Plant growth}

Seeds of wild-type barley (Hordeum vulgare L.) and the nuclear gene mutants clorina $f 2$ and clorina 104 were obtained from Professor D. Simpson (Carlsberg Research Laboratories, Copenhagen Valby, Denmark). Plants were grown in vermiculite in a growth chamber at $70 \%$ humidity in day/night regime of $10 / 14 \mathrm{~h}$ and $22 / 19^{\circ} \mathrm{C}$ at different light intensities ranging between 50 and $500 \mu \mathrm{mol} \mathrm{m}^{-2} \mathrm{~s}^{-1}$ to obtain various concentrations of chlorophyll $a$ and $b$. Etiolated leaves were grown in darkness. Primary or secondary leaves of 2- to 4-week-old plants were used for the experiments.

The grasses for the test set consisted of six $\mathrm{C}_{3}$ species (Bromus stamineus E. Desv., Danthonia caespitosa Gaudich, Lolium multiflorum Lam., Lolium perenne L., Panicum laxum Swartz., Phalaris aquatica L.), and $17 \mathrm{C}_{4}$ species (NAD-ME type: Astrebla lappacea (Lindl.) Domin, Astrebla pectinata (Lindl.) F.Muell. ex Benth., Cynondon dactylon L., Eleusine coracana Gaertn, Eragrostis superba N. M. Wolf, Panicum coloratum L., Panicum decompositum R.Br.; NADP-ME type: Bothriochloa biloba Kuntze, Bothriochloa bladhii Kuntze, Cencrus ciliaris L., Digitaria smutsi Haller, Echinochloa utilis L. Ohwi et Yabuno, Panicum antidotale Retz., Paspalum dilatatum Poir, Paspalum notatum Flügge, Pennisetum alopecuroides Rich, Pennisetum clandestinum Hochst. ex Chiov.). These test grasses were grown in 5-L pots containing sterilised garden soil supplemented with $16 \mathrm{~g}$ per pot of a controlled-release fertiliser (15/4.8/10.8/1.2 nitrogen/phosphorus/ potassium/magnesium plus trace elements boron, copper, iron, manganese, molybdenum and zinc; Osmocote Plus, Scotts,
Baulkam Hills, Australia). Plants were grown in controlled environment chambers (Phoenix, Adelaide, Australia) lit for $9 \mathrm{~h}$ with metal halide lamps supplying $1200 \mu \mathrm{mol}$ quanta $\mathrm{m}^{-2} \mathrm{~s}^{-1}$ followed by $1 \mathrm{~h}$ of incandescent lighting. Air temperature and relative humidity were maintained at $28 / 24^{\circ} \mathrm{C}$ and $60 / 80 \%$ day/ night, respectively. Fully or almost fully expanded leaves of 5- to 8-week-old plants were used for the experiment.

\section{Spectral reflectance measurements}

Spectral reflectance was measured with a spectrographic imager (CASI 601, ITRES Research Limited, Calgary, Canada) from a distance of $0.5 \mathrm{~m}$. A 2-cm long area of each attached leaf was positioned perpendicular to the imager and scanned. The imager had a spectral resolution of $\sim 1.8 \mathrm{~nm}$ (wavelength dependent) and an effective pixel size of $0.2 \times 0.2 \mathrm{~mm}^{2}$ of the leaf surface area. The leaves were irradiated with a broad-field illumination with three or six halogen lamps (50 W, Solux 4700, 36 ; EiKOLtd, Kansas City, KS, USA) from an angle of $60^{\circ}$ during spectral scans (actinic light: $540-1200 \mu \mathrm{mol} \mathrm{m}^{-2} \mathrm{~s}^{-1}$ ). Leaves were adapted to the scanning light intensity for $10 \mathrm{~min}$ before measurement. The fluorescence yield, measured with a PAM 101 (Walz, Effeltrich, Germany), reached a steady-state within this time (not shown). Reflectance spectra were obtained by calibrating the reflected signals with a white reflectance standard (Spectralon; Labsphere, North Sutton, NH, USA). Immediately after the scan, the scanned section of the leaf was excised, frozen and stored in liquid nitrogen.

The contribution of chlorophyll fluorescence to the reflectance spectrum was measured on barley with the same optical set-up as reflectance measurements, except that the illumination was provided by a different halogen lamp (15 V, $150 \mathrm{~W}, 6423$; Philips, Amsterdam, The Netherlands) fitted with two heat protection filters and a short pass filter with a cut off at $600 \pm 5 \mathrm{~nm}$ (LS-600 709K; Corion, Franklin, MA, USA). The fluorescence spectrum was measured with this short-pass filter, while the reflectance spectrum was measured without this short pass filter. The PAR was 180 and $510 \mu \mathrm{mol} \mathrm{m}^{-2} \mathrm{~s}^{-1}$, respectively, with or without the short pass filter (measured with a SKP 215, PAR; 'Quantum' sensor; Skye, Powys, UK).

\section{Pigment analyses}

Chloroplast pigments were extracted from barley leaves and quantitatively analysed according to the HPLC method of Gilmore and Yamamoto (1991). A total of 53 barley leaves were scanned and analysed (21 wild-type samples, 17 clorina f104 mutant samples and 15 clorina $f 2$ mutant samples). The chlorophyll contents of the test grasses were measured spectrometrically according to Porra et al. (1989). For each grass species of the test set, between two and five samples (one per leaf) were analysed except $P$. laxum, which only had one sample. In total there were 14 samples of $\mathrm{C}_{3}$ grasses, 18 samples of NAD-ME type $\mathrm{C}_{4}$ grasses and 23 samples of NADP-ME type grasses.

\section{Calculations}

The raw scans obtained from the CASI instrument were processed with the instrument specific software radcorr 
(ITRES Research Limited, Calgary, Canada). It applies calibration files for the spectral sensitivity of the instrument to the raw data. With the obtained data, the reflectance signal was calculated in relation to the white standard using the software ENVI 3.4+IDL 5.5 (Research Systems, Inc., Boulder, CO, USA). The image pixels belonging to the area from which the pigment analysis was performed were averaged and one reflectance curve was obtained for each leaf sample.

The CROME program was written in IDL 5.5 (Research Systems) and applied to the obtained reflectance curves and corresponding pigment data. The result was presented with Origin 7.0 (OriginLab Corporation, Northampton, MA, USA).

The curve fit in Fig. 3 and the tables were calculated with Excel 2002 (Microsoft Corporation, Redmond, WA, USA). All other graphs were plotted and fitted linearly with Origin 7.0 (OriginLab Corporation).

RGB images of the grass leaves and false colour images displaying the distribution of an index over the leaf area were calculated from spectral images and displayed with ENVI 3.4 + IDL 5.5 (Research Systems).

\section{Results}

Reflectance spectra of barley leaves with different amounts of chlorophyll a or b

The distinctive effect of chlorophyll on reflectance spectra of green foliage was illustrated by comparison of a spectrum from an etiolated barley leaf with one from wild-type barley (Fig. 1a). The etiolated barley contained carotenoids, a very low concentration of chlorophyll $a\left(0.5 \mu \mathrm{mol} \mathrm{m}^{-2}\right)$ and possibly a very low concentration of protochlorophylls, which would
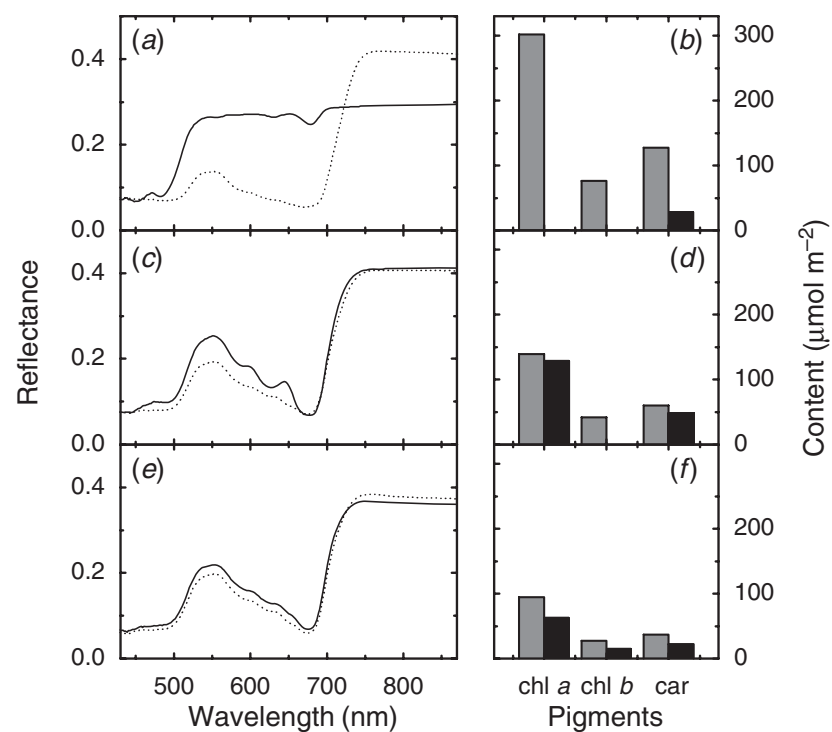

Fig. 1. Examples of reflectance spectra $(a, c, e)$ and corresponding pigment contents obtained from HPLC analysis $(b, d, f)$. The bar plots show, chlorophyll $a$ (chl $a$ ), chlorophyll $b$ (chl $b$ ) and sum of carotenoids (car). Contrasts are shown for $(a, b)$ a wild-type (dotted line, grey bars) and etiolated leaf (solid line, black bars), $(c, d)$ a wild-type (dotted line, grey bars) and clorina $f 2$ leaf (solid line, black bars) and $(e, f)$ a wild-type (dotted line, grey bars) and clorina f104 leaf (solid line, black bars). have an absorption maxima at 635 or $650 \mathrm{~nm}$. Reflectance from the etiolated leaf increased rapidly from minimal values below $500 \mathrm{~nm}$ to maximal values above $550 \mathrm{~nm}$, punctuated by a shallow trough at $677 \mathrm{~nm}$ due to absorption by chlorophyll $a$. In contrast, the reflectance spectrum was greatly modified by the presence of higher concentrations of chlorophylls $a$ and $b$ in the wild-type leaves. Reflectance from these leaves was characterised by a broad peak in green wavelengths, centred near $550-555 \mathrm{~nm}$ and a trough near $680 \mathrm{~nm}$. From 680 to $750 \mathrm{~nm}$, reflectance rapidly increased to maximal values forming a feature known as the red edge. Here, the reflectance was much higher for the wild type than the etiolated leaf.

The contribution of chlorophyll $b$ to the reflectance spectra of intact leaves was revealed by comparison of spectra from a clorina $f 2$ mutant with wild-type barley (Fig. 1c). Both samples contained similar amounts of chlorophyll $a$ (Fig. 1d) and showed a similar trough at $680 \mathrm{~nm}$ due to absorbance by chlorophyll $a$. However, at wavelengths less than $680 \mathrm{~nm}$, greater reflectance plus the appearance of an additional peak at 643-645 nm and two shoulders at 600 and $475 \mathrm{~nm}$ were consistent with less absorbance of light due to the absence of chlorophyll $b$ in the clorina $f 2$ mutant. In addition, the spectrum of this mutant showed greater reflectance in the red edge.

Finally, the reflectance spectrum from the mutant clorina f104 is shown in Fig. 1e. The reflectance peaks and shoulders caused by the reduced amount of chlorophyll $b$ were not as distinct as in clorina $f 2$.

\section{Pigment contents of leaves}

The pigment composition of leaves is a major determinant of their absorption spectra. As pigments are distributed differentially among major complexes involved in light harvesting and energy transduction in PS I and PSII, relationships between key pigments, as indicated by the slopes in Fig. 2, would be expected to differ between leaves from the barley wild type and the two mutants. First, chlorophyll $b$ is plotted $v$. chlorophyll $a$ for the barley samples (Fig. 2a). The slope of this relationship was highest in the wild type, lower in clorina f104 and zero in the chlorophyll $b$-less mutant clorina 2 (Fig. $2 a$ ). Second, lutein that is a structural component in the LHC (Kühlbrandt et al. 1994; Heinze et al. 1997) was plotted $v$. $\beta$-carotene, which occurs in the PS and inner antennae, but not the LHC (Fig. 2b). The slope of this relationship was higher in the wild type than in the mutants that have less LHC per PS (Gilmore et al. 2000). Finally, neoxanthin, which occurs only in LHCII, was plotted $v$. lutein, which occurs in the LHC of both photosystems (Lee and Thornber 1995; Paulsen 1995) (Fig. 2c). The clorina f2 mutant with the reduced amount of LHII has the lowest slope.

Chlorophyll concentrations of the grass species are summarised in Fig. $2 d$. The $\mathrm{C}_{3}$ grasses had more chlorophyll $b$ per $a$ than the $\mathrm{C}_{4}$ grasses (for chl $b: a$, ANOVA test: $P<0.0003$; Tuckey test: $P<0.01)$. However, there were no significant differences in chlorophyll composition between the NADP$\mathrm{ME}$ and NAD-ME type $\mathrm{C}_{4}$ grasses (Fig. $2 d$ ).

\section{Reflectance as a function of chlorophyll content}

The reflectance spectrum is a complex function of the scatter properties and the pigment content of the leaf. Reflectance at 

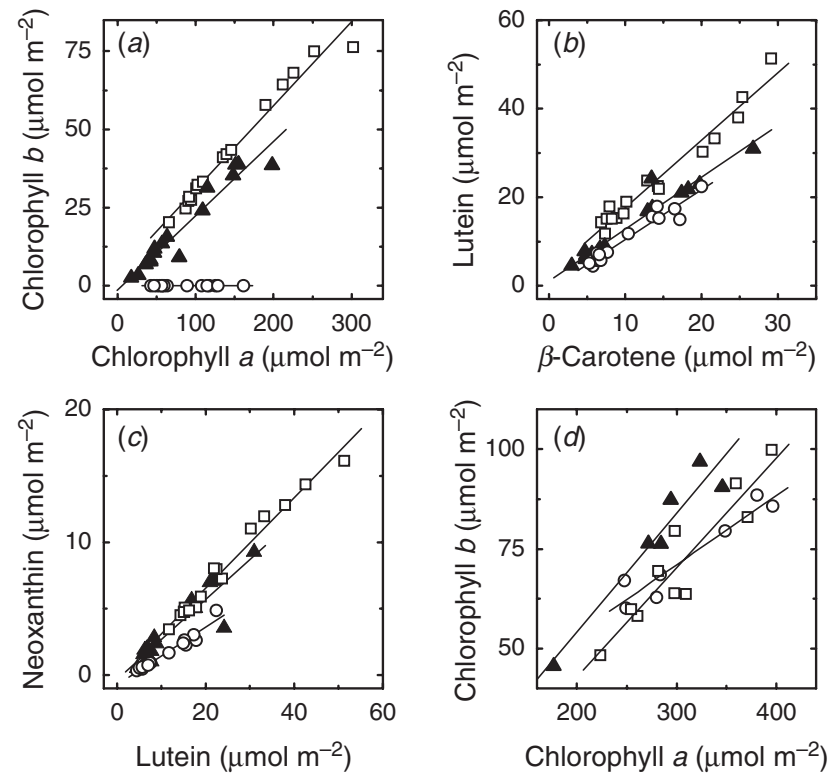

Fig. 2. Pigment contents of leaf samples from barley $(a, b, c)$ and the grass species test set $(d)$ as measured with HPLC. Chlorophyll $b v$. chlorophyll $a(a)$, lutein $v$. $\beta$-carotene $(b)$ and neoxanthin $v$. lutein $(c)$ for wild-type barley (open squares) and the two mutants clorina 104 (closed triangles) and clorina f 2 (open circles). Chlorophyll $b v$. chlorophyll $a$ for the grass species set $(d)$. Symbols indicate grasses using the $\mathrm{C}_{3}$ (closed triangles), NAD-ME type $\mathrm{C}_{4}$ (open circles) and NADP-ME type $\mathrm{C}_{4}$ photosynthetic pathways (open squares). Lines drawn by linear regression with all $r^{2}>0.85$ and $P<0.0001$ for barley $(a, b, c)$ and $r^{2}>0.93$ and $P<0.003$ for the test grass species $(d)$.

four wavelengths was plotted as a function of chlorophyll $a$ concentration to examine quantitative effects of variation in pigment content on reflectance (Fig. $3 a$ ). The wavelengths were chosen for variation in absorbance by chlorophyll $a$ with minimal effects of other pigments on the signal. Data from the barely samples were fitted to a hyperbolic function while the data from the test grasses served as test set and they fit the predicted curves reasonably well. In Fig. $3 b$, the reflectance of each sample was divided by the reflectance at $850 \mathrm{~nm}$. This normalisation decreased the variation of the data around the curves for high more than low reflectance values.

\section{Using correlation coefficients to find an equation \\ for $\mathrm{ch} / \mathrm{b}$ : a determination}

The program CROME (Davies and Fearn 2002) was used to find 'special pairs' of wavelengths that could be used to determine the chl $b: a$ ratio. CROME calculates the squared correlation for two wavelengths predicting the dependent magnitude (Fig. 4). The squared correlation is indicated with false colours at the location of two wavelengths. The correlation was generally greater for the barley (Fig. 4a) than the grass samples (Fig. 4b). This was expected because of the greater standard deviation of chl $b: a$ within the barley samples (Fig. 2). The pattern differed between the barley (Fig. 4a) and the grass species (Fig. 4b). In both sample sets, there was a correlation maximum near $637 \mathrm{~nm}$ and $627.6 \mathrm{~nm}$. From the distribution of

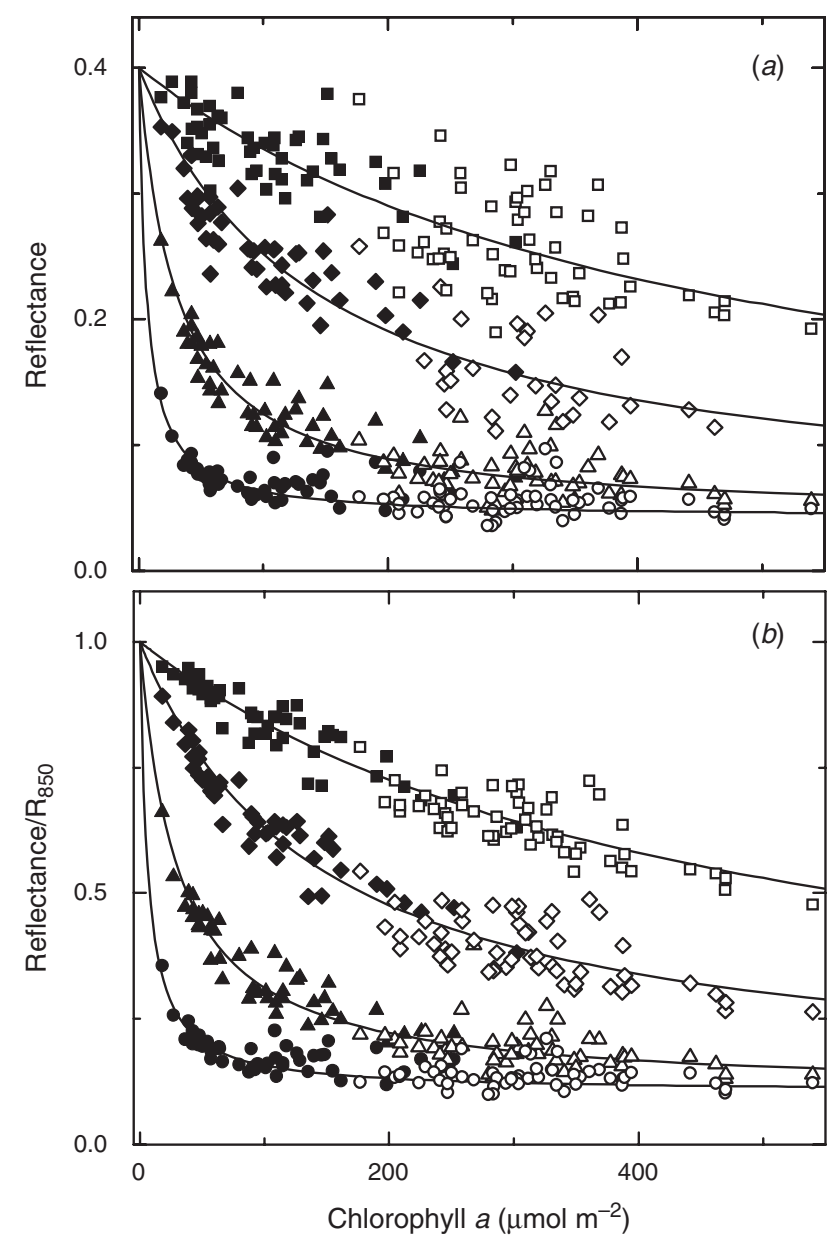

Fig. 3. Reflectance $(a)$ or normalised reflectance $(b)$ at wavelengths of $620 \mathrm{~nm}$ (triangles), $677 \mathrm{~nm}$ (circles), $706 \mathrm{~nm}$ (diamonds), $719 \mathrm{~nm}$ (squares) $v$. chlorophyll $a$ in barley (closed symbols) and the test grass samples (open symbols). Wavelengths were chosen to give a range of chlorophyll $a$ absorption where chlorophyll $b$ absorption was expected to be low. The lines are a hyperbolic functions: $y=0.4 \times(1-0.105) /($ const $\times \operatorname{chl} a+1)+0.105$ for $(a)$ and $y=(1-0.105) /($ const $\times \operatorname{chl} a+1)+0.105$ for $(b)$ The constants were 0.16 for $677 \mathrm{~nm}, 0.033$ for $620 \mathrm{~nm}, 0.0071$ for $706 \mathrm{~nm}$ and 0.0022 for $719 \mathrm{~nm}$.

the squared correlation, it can be seen that the optimal wavelength region is very narrow. The best equation found in this region is shown in Fig. $5 a$ for barley and its test for the grass species (Fig. 5b). Another long band of correlation occurred around 696-709 nm (Fig. 4). It was stronger in the grass set than in the barley set and its wavelength location was in a region where chlorophyll $b$ is unlikely to absorb light, but can affect fluorescence from chlorophyll $a$.

Effects of chlorophyll $b$ on the fluorescence emission spectrum were measured on barely leaves illuminated with blue-green light (Fig. $6 a, b$ ). The fluorescence from the wild-type barley had two fluorescence maxima, at $687 \mathrm{~nm}$ and at $738 \mathrm{~nm}$ separated by a trough, whereas this trough was missing in the clorina f 2 mutant. However, while measurable, chlorophyll fluorescence made only a small contribution to the reflectance signal of barely leaves illuminated with white light (Fig. 6c,d). 


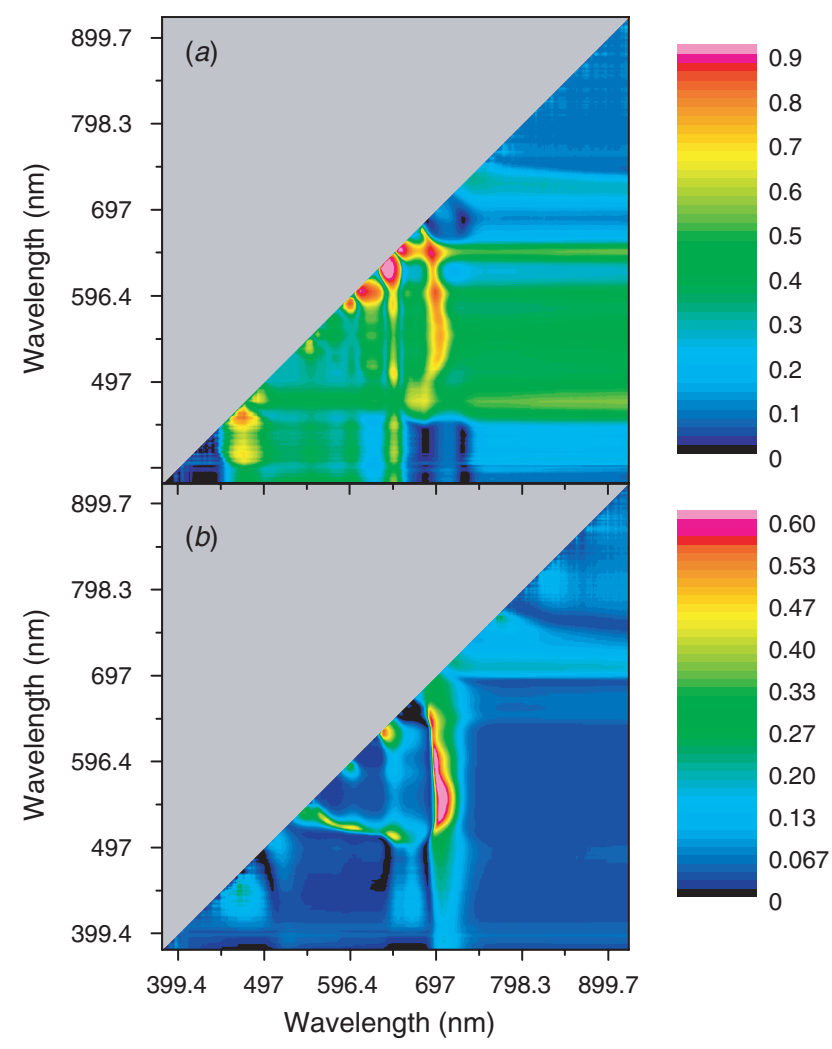

Fig. 4. CROME display (Davies and Fearn 2002) of the squared correlation coefficient (indicated by colour value; see scale) between pairs of wavelengths (indicated by location) and the chl $b: a$ ratio for barley $(a)$ and the test grass species $(b)$.

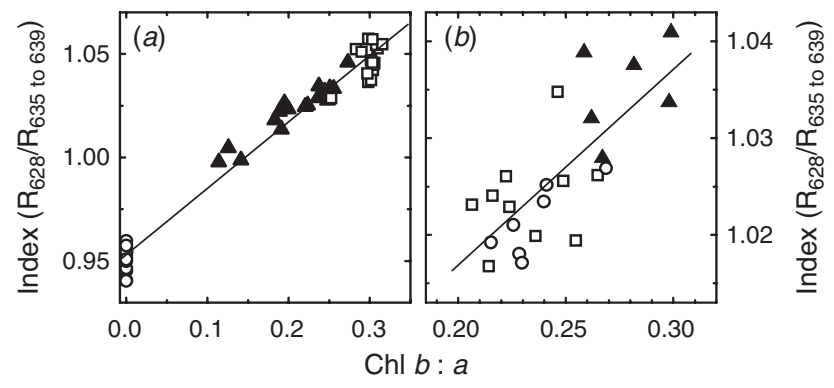

Fig. 5. Variation in the index $\mathrm{R}_{627.6} / \mathrm{R}_{(635 \text { to } 639)} v$. measured chl $b: a$ ratios for barley $(a)$ and the grass species set $(b)$. The symbols for barley $(a)$ indicate wild-type (open squares), clorina f104 (closed triangles), and clorina $f 2$ (open circles). Symbols for the grasses $(b)$ indicate species using $\mathrm{C}_{3}$ (closed triangles), NAD-ME type $\mathrm{C}_{4}$ (open circles) and NADP-ME type $\mathrm{C}_{4}$ photosynthetic pathways (open squares). Lines drawn by linear regression $(P<0.0001)$ with $r^{2}=0.98$ for $(a)$ and 0.56 for $(b)$. Note that the correlation for the grasses differs from that given in Table $\mathrm{S} 1$ because here the values for each grass species were averaged before fitting while all values were used for the calculations in Table S1.

As structural differences in the organisation of photosystems that are reflected in chlorophyll composition could affect fluorescence from chlorophyll $a$, it follows that effects of

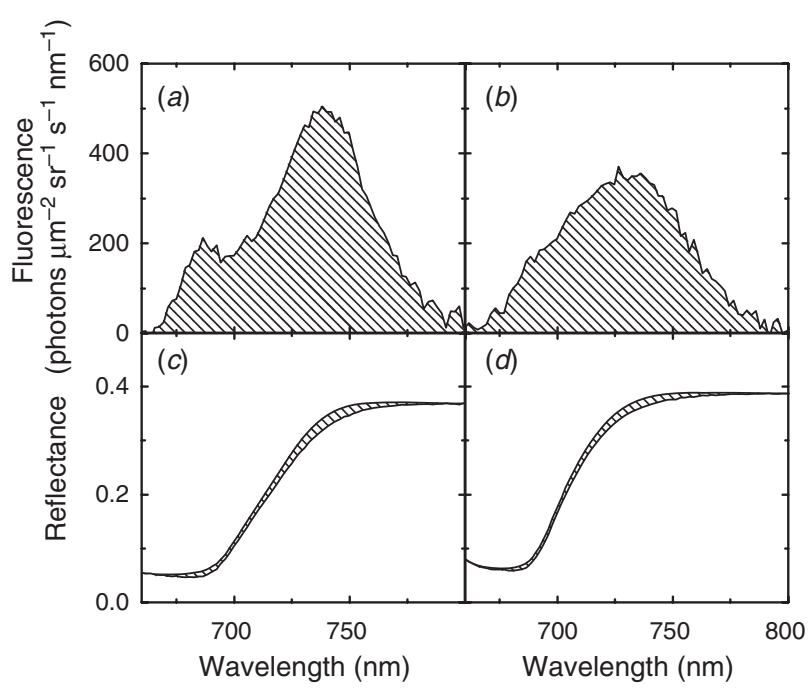

Fig. 6. Fluorescence emission spectra of a wild-type ( $a$ ) and a clorina f2 (b) barley leaf. Calculated contribution of fluorescence (hatched area) to the reflectance signal from the wild type $(c)$ and clorina $f(d)$ leaf, assuming that the yield of fluorescence remained constant between the two measurements.

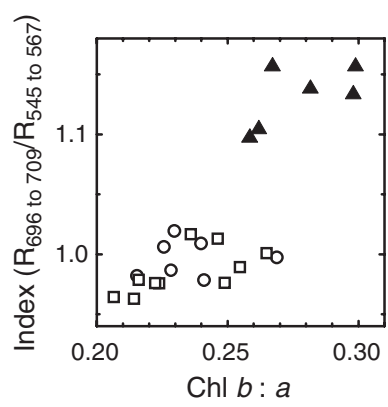

Fig. 7. $\mathrm{C}_{3}-\mathrm{C}_{4}$ index: $\mathrm{R}_{(696}$ to 709$) / \mathrm{R}_{(545}$ to 567$) v$. measured $\operatorname{chl} b: a$ ratio for the grass species set. Symbols as in Fig. $5 b$.

fluorescence on reflectance should differ between $\mathrm{C}_{3}$ and $\mathrm{C}_{4}$ species. Accordingly, an equation was found using the reflectance at wavelengths of 702 and $556 \mathrm{~nm}$ that separated $\mathrm{C}_{3}$ from $\mathrm{C}_{4}$ grasses. The significance of this separation was confirmed with a statisitical ANOVA test and a Tukey posttest (ANOVA: $P<4 \times 10^{-11}$; Tukey test: $P<10^{-9}$ ). This separation was much stronger than expected solely from differences in chlorophyll $a$ and $b$ contents and also separated $\mathrm{C}_{3}$ and $\mathrm{C}_{4}$ grasses with similar chl $b: a$ ratio in our sample set (Fig. 7). In contrast, no distinctions were found in the relationship between reflectance and chlorophyll contents between the two photosynthetic types of the $\mathrm{C}_{4}$ grasses (Fig. 7).

\section{Using the Clark method to find an equation for $\mathrm{ch} / \mathrm{b}:$ a determination}

The Clark method interprets a spectrum as a series of local features. The troughs are caused by the absorbance of a pigment in front of a continuous background (Clark and Roush 1984; Kokaly and Clark 1999; Curran et al. 2001). The background is determined by the connection between two 
extreme points in the spectrum or in the derivative of the spectrum. Figure $1 c$ showed that chlorophyll $b$ did not cause the appearance of a new feature in the reflectance spectrum, but rather, the disappearance of some small peaks between 550 and $670 \mathrm{~nm}$ in the chlorophyll $a$ reflectance spectrum. Therefore, the Clark method cannot be used in its original sense and we modified it as explained in Fig. 8. Consider the average reflectance spectrum of the clorina f2-barley without chlorophyll $b$ (Fig. 8a). The upwards line in Fig. $8 a$ (represented by a upwards arrow) depends on a feature where chlorophyll $a$ has a reflectance trough (equal to absorption peak) near $628 \mathrm{~nm}$. The connecting line from 603 to $647 \mathrm{~nm}$ is anchored at reflectance peaks (equal to absorption troughs). This feature is diminished by increase in the concentration of either chlorophyll $b$ (Fig. $8 b$ ) or chlorophyll $a$, and can also vary with differences in the scattering properties between different leaves. To correct for these factors, the upward line $\left[\mathrm{R}_{626}-0.5\right.$ $\left.\left(\mathrm{R}_{603}+\mathrm{R}_{647}\right)\right]$ was divided by the downward line $\left(\mathrm{R}_{552}-\mathrm{R}_{626}\right)$ in Fig. $8 a$. We originally also tried equations where the downward line was drawn to a connecting line between two reflectance maxima (at 552 and $710 \mathrm{~nm}$ ), but without any improvement compared with the method shown here (data not shown). If we had consequently used the Clark method, the equation would contain the factor 0.48 instead of 0.5 because the upward arrow is

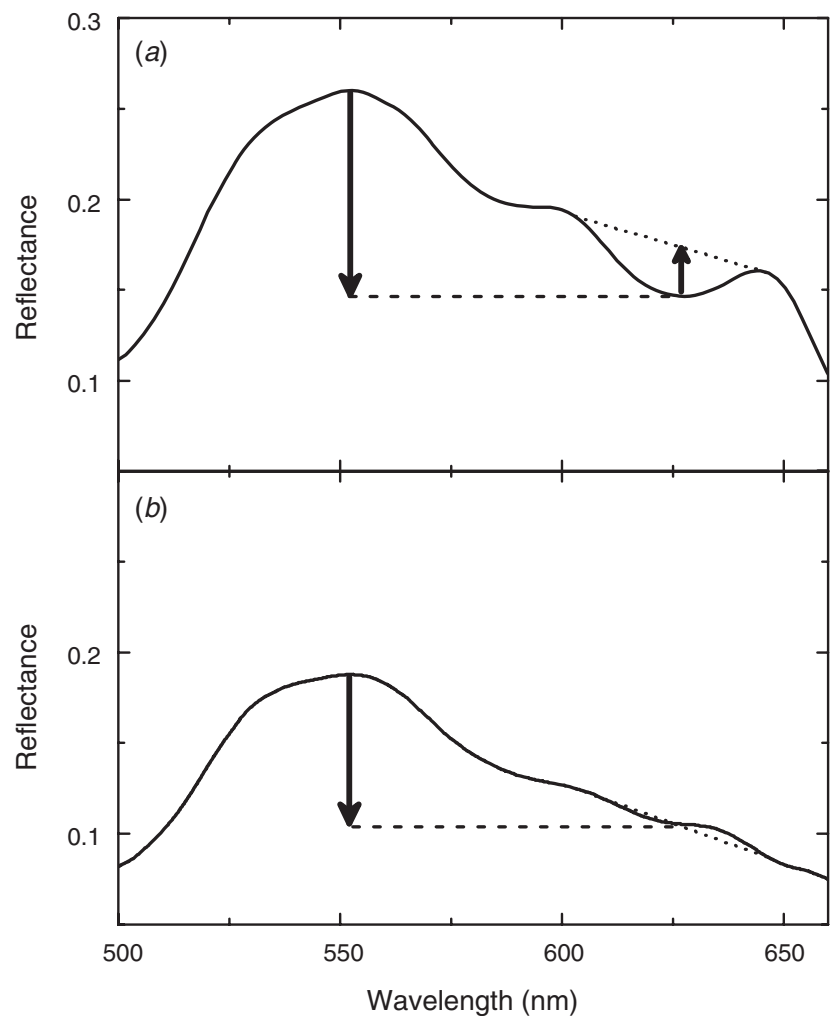

Fig. 8. Illustration of the simplified Clark method, which was used for the development of the index for $\operatorname{chl} b: a$ ratio used in Fig. 9. The curves show the average leaf reflectance of clorina 2 ( $a$ ) and wild-type barley $(b)$. The upwards arrow at $626 \mathrm{~nm}$ in panel $(a)$ indicates the magnitude in the numerator $\left[\mathrm{R}_{626}-\right.$ $\left.0.5 \times\left(\mathrm{R}_{603}+\mathrm{R}_{647}\right)\right]$ and the downwards arrow at $552 \mathrm{~nm}$ indicates the magnitude in the denominator $\left[\mathrm{R}_{552}-\mathrm{R}_{626}\right]$. not equidistant to the anchor points of the connecting line. Nevertheless, we obtained better results with a slightly bigger constant (see Table S1 available as an Accessory Publication to this paper) and therefore decided to simply use 0.5 . The result for this equation is shown in Fig. $9 a, b$ where the points are much closer to the predicted relationship than in Fig. 5. The equations derived from the Clark method were better than the equation derived from the CROME-plot.

The equations developed in the present study were used to create maps of the chl $b$ : $a$ ratio and the $\mathrm{C}_{3}-\mathrm{C}_{4}$ index from spectral scans of different grass leaves, as shown in Fig. 10. Maps created with the equation for the $\operatorname{chl} b: a$ determination are displayed for barley (Fig. 10b) and three grasses of the genus Panicum, which have three different types of photosynthesis (Fig. 10d). Calculations used the fitted line for the grass species set (Fig. 9) because their chlorophyll concentrations were closer to natural values than the barley samples. As a result the obtained values for the barley mutants were a bit too low. Maps of the $\mathrm{C}_{3}-\mathrm{C}_{4}$ index are displayed in Fig. 10e. Positive and negative values indicate $\mathrm{C}_{3}$ and $\mathrm{C}_{4}$ grasses, respectively. $P$. laxum, the $\mathrm{C}_{3}$ grass is positive, while negative values were obtained for $P$. coloratum and $P$. antidotale. Only points with an $\mathrm{NDVI}_{680}$ higher than 0.5 were included in the calculation. Therefore, the white stripe in $P$ coloratum was not included in the calculation and set to zero. Except for the midrib in $P$. antidotale where the chl $a: b$ ratio appears higher than in the surrounding lamina, there were no spatial patterns in the distribution of either index over the leaf surfaces.

\section{Comparison with literature}

Table S1 summarises equations from the literature and equations developed in the present study, their suggested purpose and the correlation coefficient with the measured chl $b: a$ ratio. The equation from Yoder and Daley (1989) had a good correlation with our dataset.

Table S2 shows the squared correlation coefficient and a sensitivity value (Gitelson et al. 2002) for the different subsets. A criterion for the evaluation of an equation is not

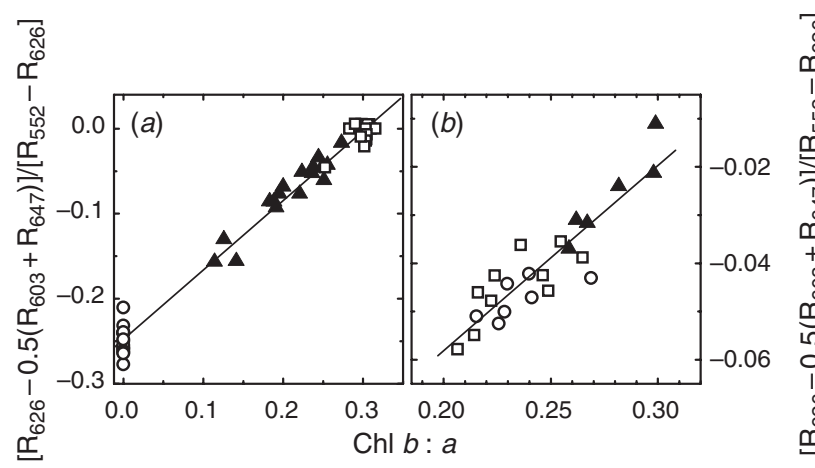

Fig. 9. Variation in the chl $b: a$ index $\left[\mathrm{R}_{626}-0.5 \times\left(\mathrm{R}_{603}+\mathrm{R}_{647}\right)\right] /\left[\mathrm{R}_{552}-\right.$ $\mathrm{R}_{626}$ ] as a function of the chl $b: a$ ratio measured in the barley $(a)$ and grass species set $(b)$. Symbols are as in Fig. 5. Lines drawn by linear regression $(P<0.0001)$ with $r^{2}=0.98$ for $(a)$ and 0.81 for $(b)$. Note that the correlation for the grasses differs from that given in Table $\mathrm{S} 1$ because here the values for each grass species were averaged before fitting while all values were used for the calculations in Table S2. Also, the constant c1 in Table S1 was set to 0.5 here. 


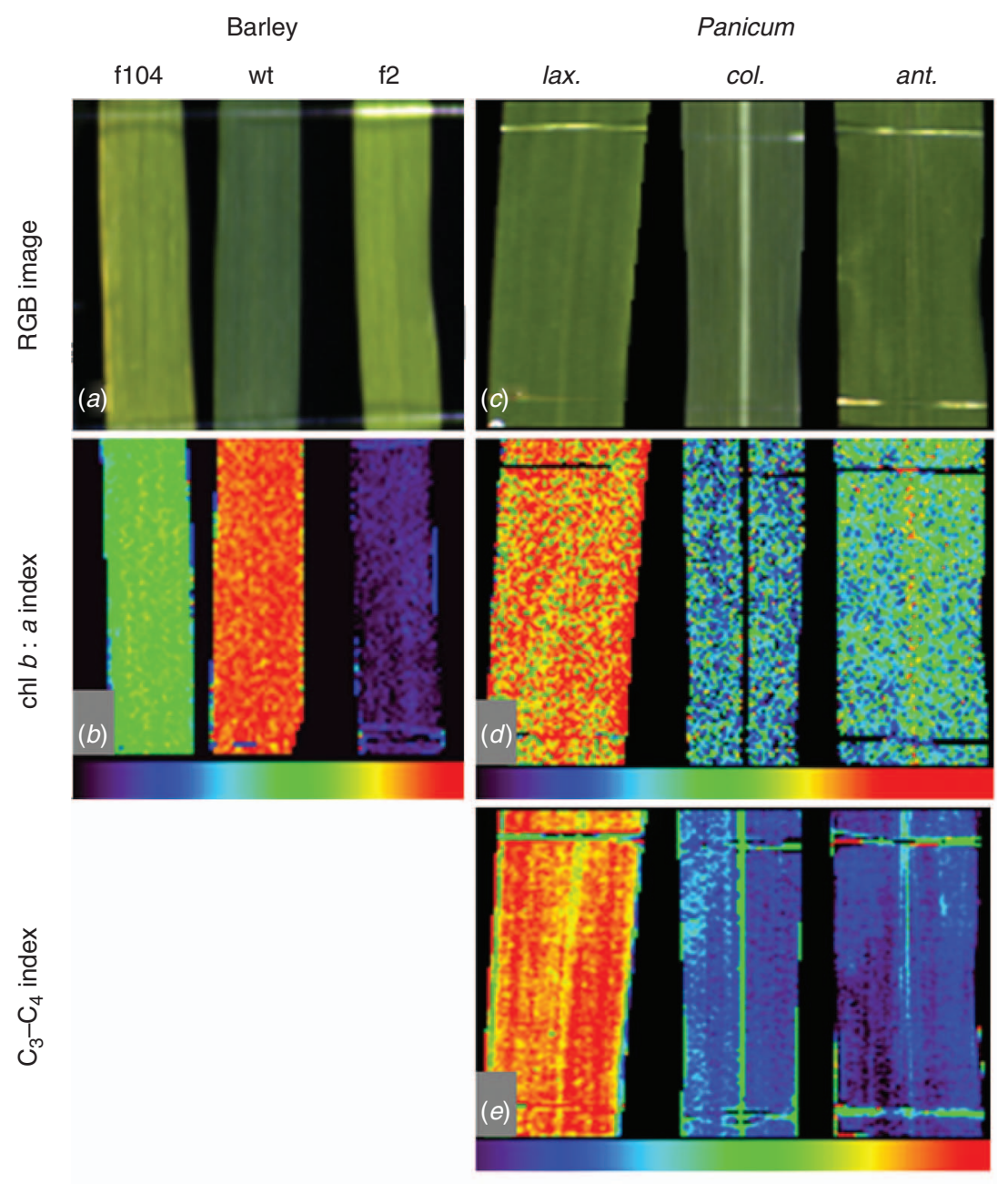

Fig. 10. RGB images of barley $(a)$ and test grasses $(c)$ and corresponding images created with the chl $b: \mathrm{a}(b, d)$ and $\mathrm{C}_{3}-\mathrm{C}_{4}$ indices (e). Images are shown for barley clorina f104 (f104), wild-type (wt) and clorina $f 2$ (f2) and three species of Panicum differing in photosynthetic pathway: P. laxum $\left(\mathrm{C}_{3}\right)$, $P$. coloratum (NAD-ME type $\mathrm{C}_{4}$ ), and $P$. antidotale (NADP-ME type $\mathrm{C}_{4}$ ). Chl $b: a$ index: $\left[\mathrm{R}_{626}-\right.$ $\left.0.5 \times\left(\mathrm{R}_{603}+\mathrm{R}_{647}\right)\right] /\left[\mathrm{R}_{552}-\mathrm{R}_{626}\right] \times 1.64+0.31(b)$ with scale from -0.1 to 0.3 , and $(d)$ with scale from 0.18 to 0.3$) . C_{3}-C_{4}$ index: $R_{(696}$ to 709$) / R_{(545}$ to 567$)-1.05$ (e) with scale from -0.1 to 0.1 ).

only how well it correlates with the measured pigment, but also how little it cross-correlates with another pigment as indicated by the sensitivity value in Table S2. The optimal sensitivity for the total chlorophyll determination would be ' 1 ' for all subsets, meaning that it depends equally on both pigments in all subsets. A low sensitivity indicates a good equation for chlorophyll $a$ determination, while a high sensitivity indicates a good equation for chlorophyll $b$ determination. The results for the sensitivity are strongly dependent on the sample set. Some of the differences are due to systematic errors that are caused by the sub-samples having different offsets for the relationship between chlorophyll $b$ and $a$ (Fig. 2).

Different equations achieved the best squared correlation for chlorophyll $a$ or total chlorophyll determination in either the barley or the grass species set. The last line demonstrates that a lot of variation was introduced by choosing a sample set with a high variance in chl $b: a$ ratio. If we added a correction to the index for total chlorophyll in grasses by simply adding the best chl $b: a$ index from Table S1 (Fig. 9), then a much better correlation to chlorophyll $a, b$ or total chlorophyll was achieved with both sample sets. When all samples were pooled together to find the best index for chlorophyll determination, the green region $\left(\mathrm{R}_{850}\right.$ to $860 / \mathrm{R}_{540}$ to 560 ) showed the highest squared correlation for total chlorophyll (0.90), chlorophyll $a(0.89)$ or chlorophyll $b$ (0.85) determination.

\section{Discussion}

The results of the present study show how chlorophylls $a$ and $b$ differentially and interactively affect the reflectance spectra of leaves and, conversely, how this knowledge can be used for remote quantitative measurement of chlorophyll composition and remote detection of $\mathrm{C}_{3}$ and $\mathrm{C}_{4}$ grasses, as discussed below.

We first examined how the relative pigment content of the barley leaves was influenced by the mutations (Fig. 2). The pigment compositions were consistent with previously 
published characterisations of photosynthetic attributes of barley and the two barley mutants (Gilmore et al. 2000). This gives us confidence in using data in the literature to interpret spectral features identified in the present study.

Pigments are distributed differently among major complexes and chlorophyll $b$ occurs only in LHCs. The relationship between chlorophyll $b: a$ is therefore commonly used as an indicator for the amount of LHCs per PS although this is complicated by the fact that LHCIIs contain more chl $b: a$ than LHCIs. In contrast to chlorophyll, carotenoids can also occur dissolved within the thylakoid membranes (Morosinotto et al. 2003). Therefore, relationships between carotenoids would not necessarily be expected to correlate with the LHC:PS ratio. Nevertheless, our results showed that the relationship between lutein and $\beta$-carotene indicates the LHC:PS ratio and likewise the relationship between neoxanthin and lutein indicates the LHCI:LHCII ratio in the leaf. Despite these results, we did not attempt to use the blue region of the reflectance spectrum to gain information about our samples, mainly because both chlorophylls and all six main carotenoids absorb in this region (Lichtenthaler 1987). The overall absorbance is very strong, making it unlikely to provide accurate information about pigment composition because of saturation of the signal.

We used the variation in chlorophyll contents of leaves from barley mutants to assess reflectance spectra. The etiolated leaves had a spectrum that was similar to that obtained from senescing maple leaves with low chlorophyll $a$ content (Gitelson and Merzlyak 1994). In the absence of pigments, the reflected light mainly results from light scattering within the leaf caused by refraction at air-water boundaries, cell walls and membranes (Heber 1969; Grant 1987). The shape of the etiolated leaf reflectance above $550 \mathrm{~nm}$ (Fig. 1) gave no indication of variation in scatter with wavelength except for a slight steady increase with longer wavelengths. Similarly, Rühle and Wild (1979) found constant light dispersion within leaves between 550 and $800 \mathrm{~nm}$.

The reflectance in the infrared region was higher for the wild type and mutants than for the etiolated leaves (Fig. 1) indicating greater scattering, mainly due to leaf thickness. In the presence of pigments, light absorption influences the path length of scattered light; the resulting reflectance spectrum is a complex function of the scatter properties and the pigment content of the leaf. Nevertheless, simple indices have been used with good success for the determination of chlorophyll content of leaves from reflectance spectra (Table S2). The most successful indices (highest correlation in Table S2) for chlorophyll determination contain reflectance in the NIR, where there is little absorption and reflectance at a wavelength weakly absorbed by chlorophyll (c.f. Gitelson and Merzlyak 1997)

As shown in Fig. 3, the dependence of reflectance on pigment concentration can be described empirically with a hyperbolic function:

$$
R=\frac{R_{0}-R_{\text {surf }}}{\text { const } \times \mathrm{Abs}+1}+R_{\text {surf }} \Leftrightarrow \mathrm{Abs}=\frac{1}{\text { const }}\left(\frac{R_{0}-R_{\text {surf }}}{R-R_{\text {surf }}}-1\right)
$$

With $R_{0}$ for a reflectance with no absorption (Abs), which implies that it solely depends on the scatter properties of the leaf and its surface reflectance $\left(R_{\text {Surf }}\right)$.

If absorbance is zero, Eqn 1 simplifies to:

$$
R=R_{0}
$$

If the Absorbance within the leaf is infinite, the reflectance of the leaf depends only on the properties of the leaf surface.

$$
R=R_{\text {surf }}
$$

If $R_{\text {Surf }}$ is zero, Eqn 1 simplifies to:

$$
\mathrm{Abs}=\frac{1}{\mathrm{const}}\left(\frac{R_{0}}{R}-1\right)
$$

If $R_{\text {Surf }}$ is small in comparison to $R$ and $R_{0}$, Eqn 1 can be simplified to:

$$
\mathrm{Abs} \approx a \times \frac{R_{0}}{R}-b
$$

When $R_{0}$ is replaced by $R_{\mathrm{NIR}}$ (reflectance in the near infrared), this equation becomes a simple index. It implies that the scatter properties in the near infrared are equal, or at least proportional, to the scatter properties at the wavelength of interest.

The optimal wavelength for calculating chlorophyll $a$ concentration in a given sample set depends on its average chlorophyll content because that determines which wavelength has the optimal variance in the reflectance index (Fig. 3). For our barley sample set, which had an average chlorophyll $a$ content of $104 \mu \mathrm{mol} \mathrm{m} \mathrm{s}^{-2}$, variance was optimal between 704 and $713 \mathrm{~nm}$ (Table S2). For the grass samples with a much higher chlorophyll $a$ content (Table S2), the optimum was at a wavelength with a lower absorption coefficient at 733 to $738 \mathrm{~nm}$. In general, the higher the chlorophyll content of the sample, the lower the absorption coefficient of the chosen wavelength should be. Since the chlorophyll content of leaves cannot be diluted to reach an optimal concentration for spectral analysis, chlorophyll $a$ determination can be optimised by using an appropriate wavelength. However, this procedure is complicated by two factors: (i) different sample sets may vary in their scatter properties and (ii) there is a contribution to the reflectance spectrum from chlorophyll $a$ fluorescence in the red edge.

Chlorophyll $b$ is more difficult to determine than chlorophyll $a$ because it needs to be determined against a background of a high chlorophyll $a$ concentration. We found that the best equation for determination of chlorophyll $b$ used wavelengths in the green region of the spectrum (Table S2) and was better correlated with chlorophyll $a$ than to chlorophyll $b$ for the grass species set. Consistent with results for both chlorophyll $a$ and total chlorophyll, we obtained a different optimal equation for each sample set (Table S2).

Since our samples were chosen to have a high variation in chl $b: a$ ratios, the absolute determination of chlorophyll $a, b$ or total chlorophyll was difficult. The different relationships between chlorophyll $a$ and $b$ for the sub-sets introduced a systematic error that led to systematic differences between the sensitivity coefficients. Therefore, a good balance of sensitivity 
(sensitivity coefficient close to one in Table S2) between chlorophyll $a$ and $b$ within one sample sub-set is not necessarily observed for another sample sub-set. The slope of the index for total chlorophyll was different for the $\mathrm{C}_{3}$ and $\mathrm{C}_{4}$ grasses (not shown). This systematic error could be corrected by adding the chl $b: a$ index from Table S1 to the best equation in Table S2. Nevertheless, this correction required the introduction of a different parameter for each sample set and chlorophyll. Therefore, this idea is more a demonstration of the strong influence of the $\operatorname{chl} b: a$ ratio on the chlorophyll determination than a solution for the problem.

\section{CROME approach v. Clark method}

We used a statistical approach (CROME; Davies and Fearn 2002) to find optimal wavelengths for the determination of the chl $b: a$ ratio. With the grass sample set, we confirmed that the obtained equation was well correlated with the chl $b: a$ ratio (Fig. 5). Nevertheless, using just observation and the Clark idea, we obtained a much better equation (Fig. 9), as indicated by statistics. The second approach has the advantage that it does not use the same statics in the development as well as in the test of the hypothesis. Despite this drawback, the CROME approach led to an equation that clearly separated the $\mathrm{C}_{3}$ and $\mathrm{C}_{4}$ plants of our grass species set (Fig. 7). This separation was stronger than expected from the differences in chl $b: a$ ratio.

\section{Interrelation between chl b : a and fluorescence signals in the red edge}

The fluorescence spectrum measured with the CASI system on the barley wild type (Fig. 5) was consistent with previous observations with time resolved spectral fluorescence measurements showing a fluorescence band at $685 \mathrm{~nm}$ (attributed to PSII) and at 705 and $740 \mathrm{~nm}$ (attributed to PSI) (Gilmore et al. 2000). The fluorescence at $685 \mathrm{~nm}$ was generally higher in the cited study than here. This was most probably because their samples were dark-adapted and water-infiltrated, while ours were light-adapted and untreated. In the clorina $f 2$ mutants, Gilmore et al. (2000) observed a strong fluorescence band at $710 \mathrm{~nm}$. This result is in agreement with our observation that the trough, which was located between the two fluorescence peaks in the wild type, disappeared in the clorina $f 2$ mutant. The appearance of the strong $710 \mathrm{~nm}$ band is caused by profound structural changes influencing the energy transfer to PSI in the clorina $f 2$ mutant (Gilmore et al. 2000). The contribution of fluorescence to the reflectance spectrum we obtained here for the wild type (Fig. $6 d$ ) was similar to the findings of Zarco-Tejada et al. (2000). The changes in fluorescence are different between the two mutants (Gilmore et al. 2000).

The index we found for the separation of $\mathrm{C}_{3}$ and $\mathrm{C}_{4}$ grasses contained both a green waveband $(556 \mathrm{~nm})$ and a waveband in the region of chlorophyll $a$ fluorescence (702 nm) (Fig. 7). Changes in the fluorescence spectra correlating with changes in the chl $a: b$ ratio have been observed before with plants of the genus Flaveria having $\mathrm{C}_{3}, \mathrm{C}_{3}-\mathrm{C}_{4}$ intermediates and $\mathrm{C}_{4}$-like and $\mathrm{C}_{4}$ photosynthesis (Pfündel and Pfeffer 1997) or purified mesophyll and bundle-sheaths thylakoids from various $\mathrm{C}_{4}$ and $\mathrm{C}_{3}$ plants (Pfündel et al. 1996). Again, changes in the energy transfer between photosynthetic complexes are correlated with the changes in the chl $a: b$ ratio.

Another example is the index used by Nicotra et al. (2003). They found changes in the fluorescence region of the reflectance spectrum accompanied by changes in the chl $a: b$ ratio due to frost in Eucalyptus pauciflora Sieb. ex Spreng leaves. While they could use those changes in the fluorescence region to determine chl $a: b$ ratios in their study, the same equation did not work here.

In all three examples, changes in chl $b: a$ ratio were accompanied by changes in the fluorescence region of the reflectance spectrum. However, the fluorescence changes were not the same because the biochemical mechanisms affecting changes in the fluorescence emissions were also not the same. Therefore, an equation found for the determination of chlorophyll ratios on one system could not be transferred to another system when it included fluorescence bands. However, when all samples were pooled together, an equation using wavebands from the green region (where chlorophyll absorbs weakly and there are no contributions to the spectrum from fluorescence), showed the best squared correlation for absolute chlorophyll determination.

\section{Future application}

The equation suggested here (Fig. 9) for the determination of chl $b: a$ ratio has the advantage that it does not include wavebands with strong chlorophyll fluorescence and works well on all tested grasses. This equation might be applicable in remote sensing because the $\operatorname{chl} b: a$ ratio has been shown to change in plants with various changes in environmental conditions like sun shade adaptation (Boardman 1977), nitrogen availability (Kitajima and Hogan 2003) and frost adaptation (Nicotra et al. 2003).

The equation suggested here for separation of $C_{3}$ and $C_{4}$ grasses is based on contributions of chlorophyll fluorescence to the reflectance spectrum and might find application in global change biology. For example, as $\mathrm{C}_{4}$ photosynthesis is an adaptation to warmer climates and low atmospheric $\mathrm{CO}_{2}$ concentrations (von Caemmerer 2000), changes in the geographic distribution of $\mathrm{C}_{4}$ grasses may occur in response to global climate warming (Sage and Kubien 2007). Our study might improve algorithms used to monitor those changes with remote sensing.

\section{Acknowledgements}

We thank Professors Terry Caelli, Paul Kriedemann and Jochen Zeil and Drs Adam Gilmore and Adrienne Nicotra for critical discussion of previous versions of the manuscript, Dr Oula Ghannoum for providing $\mathrm{C}_{4}$ grass seeds, Dr David Barker for pigment determinations, Drs Luigi Ranzullo, Glenn Newnham and Susanne Thulin for inspiring discussions.

\section{References}

Boardman NK (1977) Comparative photosynthesis of sun and shade plants. Annual Review of Plant Physiology 28, 355-377. doi: 10.1146/annurev. pp.28.060177.002035

Bossman B, Knoetzel J, Jansson S (1997) Screening of clorina mutants of barley (Hordeum vulgare L.) with antibodies against light-harvesting proteins of PSI and PSII: absence of specific antenna proteins. Photosynthesis Research 40, 287-294.

Clark RN, Roush TL (1984) Reflectance spectroscopy: quantitative analysis techniques for remote sensing applications. Journal of Geophysical Research 89, 6329-6340. doi: 10.1029/JB089iB07p06329 
Curran PJ, Dungan JL, Peterson DL (2001) Estimating the foliar biochemical concentration of leaves with reflectance spectrometry. Testing the Kokaly and Clark methodologies. Remote Sensing of Environment 76, 349-359. doi: 10.1016/S0034-4257(01)00182-1

Davies AMC, Fearn T (2002) Doing it faster and smarter (Lesson 6 of matrix algebra). Spectroscopy Europe 14, 24-26.

Edwards G, Walker D (1983) ' $\mathrm{C}_{3}, \mathrm{C}_{4}$ : mechanisms, and cellular and environmental regulation of photosynthesis.' (Blackwell Scientific Publications: Oxford, UK)

Ghannoum O, Evans JR, Chow WS, Andrews TJ, Conroy JP, von Caemmerer $S$ (2005) Faster Rubisco is the key to superior nitrogen-use efficiency in NADP-malic enzyme relative to NAD-malic enzyme $\mathrm{C}_{4}$ grasses. Plant Physiology 137, 638-650. doi: 10.1104/pp.104.054759

Gilmore AM, Yamamoto HY (1991) Resolution of lutein and zeaxanthin using a lightly carbon-loaded $\mathrm{C} 18$ high performance liquid chromatographic column. Journal of Chromatography. A 543, 137-145. doi: 10.1016/S0021-9673(01)95762-0

Gilmore AM, Itoh S, Govindjee (2000) Global spectral-kinetic analysis of room temperature chlorophyll $a$ fluorescence from light-harvesting antenna mutants of barley. Philosophical Transactions of the Royal Society of London. Series B, Biological Sciences 355, 1371-1384. doi: 10.1098/rstb.2000.0699

Gitelson AA, Merzlyak MN (1994) Quantitative estimation of chlorophyll- $a$ using reflectance spectra: experiments with autumn chestnut and maple leaves. Journal of Photochemistry and Photobiology. B, Biology 22, 247-252. doi: 10.1016/1011-1344(93)06963-4

Gitelson AA, Merzlyak MN (1997) Remote estimation of chlorophyll content in higher plants. International Journal of Remote Sensing 18(12), 2691-2697. doi: 10.1080/014311697217558

Gitelson AA, Zur Y, Chivkunova OB, Merzlyak MN (2002) Assessing carotenoid content in plant leaves with reflectance spectroscopy. Photochemistry and Photobiology 75, 272-281. doi: 10.1562/00318655(2002)075<0272:ACCIPL $>2.0 . C O ; 2$

Grant L (1987) Diffuse and specular characteristics of leaf reflectance. Remote Sensing of Environment 22, 309-322. doi: 10.1016/0034-4257(87) 90064-2

Green BR, Durnford DG (1996) The chlorophyll-carotenoid proteins of oxygenic photosynthesis. Annual Review of Plant Physiology and Plant Molecular Biology 47, 685-714. doi: 10.1146/annurev. arplant.47.1.685

Harrison MA, Nemson JA, Melis A (1993) Assembly and composition of the chlorophyll $a-b$ light harvesting complex of barley (Hordeum vulgare L.): immunochemical analysis of chlorophyll $b$-less and chlorophyll $b$ deficient mutants. Photosynthesis Research 38, 141-151. doi: 10.1007/ BF00146413

Hatch MD (1987) $C_{4}$ photosynthesis: a unique blend of modified biochemistry, anatomy and ultrastructure. Biochimica et Biophysica Acta 895, 81-106.

Heber U (1969) Conformational changes of chloroplasts induced by illumination of leaves in vivo. Biochimica et Biophysica Acta 180, 302-319. doi: 10.1016/0005-2728(69)90116-9

Heinze I, Pfündel E, Hühn M, Dau H (1997) Assembly of light harvesting complexes II (LHC-II) in the absence of lutein - a study on the acarotenoid-free mutant C-2A'-34 of the green alga Scenedesmus obliquus. Biochimica et Biophysica Acta 1320, 188-194. doi: 10.1016/ S0005-2728(97)00022-4

Kitajima K, Hogan KP (2003) Increases of chlorophyll $a / b$ ratios during acclimation of tropical woody seedlings to nitrogen limitation and high light. Plant, Cell \& Environment 26(6), 857-865. doi: 10.1046/j.13653040.2003.01017.x
Knoetzel J, Simpson D (1991) Expression and organisation of antenna proteins in the light- and temperature-sensitive barley mutant clorina f104. Planta 185, 111-123. doi: 10.1007/BF00194522

Kokaly RF, Clark RN (1999) Spectroscopic determination of leaf biochemistry using band-depth analysis of absorption features and stepwise multiple linear regression. Remote Sensing of Environment 67, 267-287. doi: 10.1016/S0034-4257(98)00084-4

Kühlbrandt W, Wang DA, Fujiyoshi Y (1994) Atomic model of plant lightharvesting complex. Nature 367, 614-621. doi: 10.1038/367614a0

Lee AL-C, Thornber JP (1995) Analysis of the pigment stoichiometry of pigment-protein complexes from barley (Hordeum vulgare). Plant Physiology 107, 565-574. doi: 10.1104/pp.107.2.565

Lichtenthaler H (1987) Chlorophylls and carotenoids: pigments of photosynthetic biomembranes. Methods in Enzymology 148, 350-382. doi: 10.1016/0076-6879(87)48036-1

Morosinotto T, Caffarri S, Dall'Osto L, Bassi R (2003) Mechanistic aspects of the xanthophyll dynamics in higher plant thylakoids. Physiologia Plantarum 119, 347-354. doi: 10.1034/j.1399-3054.2003.00213.x

Nicotra AB, Hofmann M, Siebke K, Ball MC (2003) Spatial patterning of pigmentation in evergreen leaves in response to freezing stress. Plant, Cell \& Environment 26, 1893-1904. doi: 10.1046/j.1365-3040.2003.01106.x

Paulsen H (1995) Chlorophyll a/b-binding proteins. Photochemistry and Photobiology 62(3), 367-382. doi: 10.1111/j.1751-1097.1995.tb02357.x

Pfündel E, Pfeffer M (1997) Modification of photosystem I light harvesting of bundle-sheath chloroplasts occurred during the evolution of NADP-malic enzyme $\mathrm{C}_{4}$ photosynthesis. Plant Physiology 114, 145-152.

Pfündel E, Nagel E, Meister A (1996) Analyzing the light energy distribution in the photosynthetic apparatus of $\mathrm{C}_{4}$ plants using highly purified mesophyll and bundle sheath thylakoids. Plant Physiology 112, $1055-1070$.

Porra RJ, Thompson WA, Kriedemann PE (1989) Determination of accurate extinction coefficients and simultaneous equations for assaying chlorophylls $a$ and $b$ extracted with four different solvents: verification of the concentration of chlorophyll standards by atomic absorption spectroscopy. Biochimica et Biophysica Acta 975, 384-394. doi: 10.1016/S0005-2728(89)80347-0

Rühle W, Wild A (1979) The intensification of absorbance changes in leaves by light-dispersion. Planta 146, 551-557. doi: 10.1007/BF00388831

Sage RF, Kubien DS (2007) The temperature response of $\mathrm{C}_{3}$ and $\mathrm{C}_{4}$ photosynthesis. Plant, Cell \& Environment 30, 1086-1106. doi: $10.1111 / \mathrm{j} .1365-3040.2007 .01682 . \mathrm{x}$

von Caemmerer S (2000) 'Biochemical models of leaf photosynthesis.' (CSIRO Publishing: Collingwood, Australia)

Yoder BJ, Daley LS (1989) Development of a visible spectroscopic method for determining chlorophyll $a$ and $b$ in vivo in leaf samples. Spectroscopy 5, 44-50.

Zarco-Tejada PJ, Miller JR, Mohammed GH, Noland TL (2000) Chlorophyll fluorescence effects on vegetation apparent reflectance: I. Leaf-level measurements and model simulation. Remote Sensing of Environment 74(3), 582-595. doi: 10.1016/S0034-4257(00)00148-6

Manuscript received 29 July 2009, accepted 28 September 2009 